\title{
Polarity-dependent cerebellar transcranial direct current stimulation modulates online functions of the alerting attention network
}

Aysha N. Kinakool, BPT, MScNPT, Turki S. Abualait, PT, PhD.

\begin{abstract}
الأهداف : دراسة التأثير التعديلي القطبي، المهبطي أو الوهمي التهبي

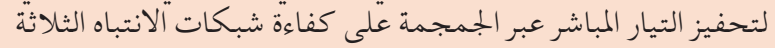

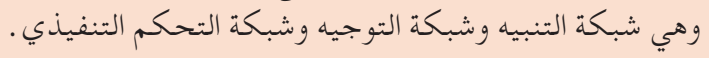

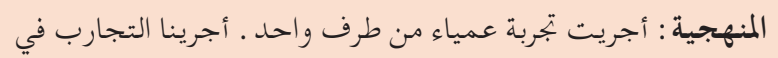

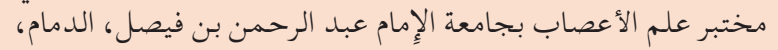

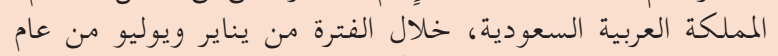

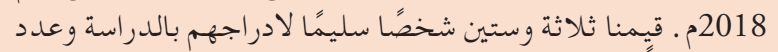

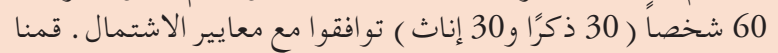

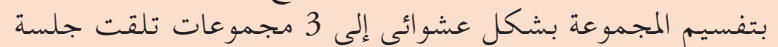

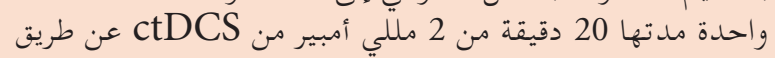

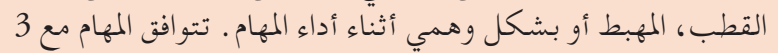

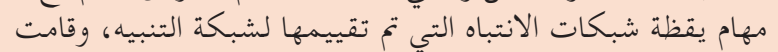

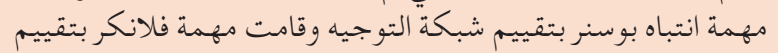

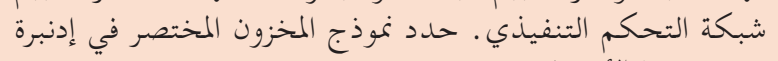
مدى يدوية الأشخاص . ملكم التفيدي.

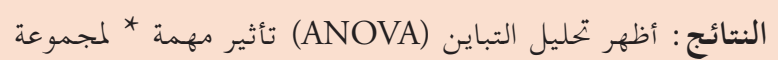

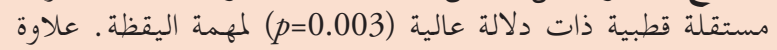

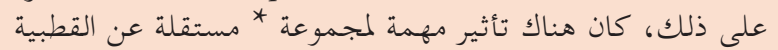

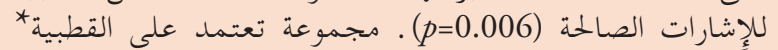

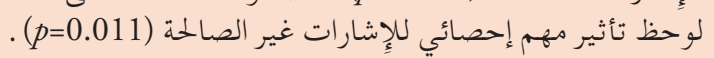

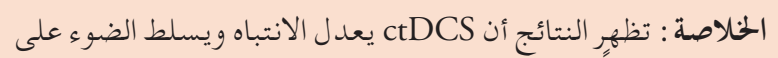

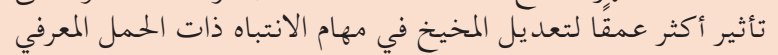
العالي أنير أكنر
\end{abstract}

Objectives: To investigate the modulatory effect of anodal, cathodal and sham cerebellar transcranial direct current stimulation (ctDCS) on the efficiency of the 3 attention networks namely alerting network, orienting network, and the network of executive control.

Methods: A sham-controlled, single-blind study. Experiments were performed at the neuroscience lab of Imam Abdulrahman Bin Faisal University, Dammam, Kingdom of Saudi Arabia, between the months of January-July of 2018. Sixty-three healthy subjects were assessed for eligibility and a total of 60 subjects ( 30 males and 30 females) met the inclusion criteria. Subjects were randomly divided into 3 groups that received a single 20 -minute session of $2 \mathrm{~mA}$ of anodal, cathodal or sham ctDCS while performing tasks. The tasks corresponded to the 3 attention networks-vigilance task assessed the alerting network, Posner's attention task assessed the orienting network and Flanker task assessed the network of executive control. The Edinburgh handedness inventory-short form determined the subjects' handedness.

Results: Analysis of variance (ANOVA) showed a highly significant polarity-independent group*task effect $(p=0.003)$ for the vigilance task. Moreover, there was a significant polarity-independent group*task effect for valid cues $(p=0.006)$. A polarity-dependent group*task effect was observed for invalid cues $(p=0.011)$.

Conclusion: Findings demonstrate that ctDCS modulates alerting attention and highlights a more profound effect of cerebellar modulation in attention tasks with higher cognitive load.

Neurosciences 2021; Vol. 26 (4): 323-330 doi: 10.17712/nsj.2021.4.20210013

From the Department of Physical Therapy (Kinakool, Abualait), College of Applied Medical Sciences, Imam Abdulrahman Bin Faisal University, Dammam, Kingdom of Saudi Arabia, and from the Department of Psychology (Kinakool), York University, Toronto, Canada.

Received 25th January 2021. Accepted 11th July 2021.

Address correspondence and reprint request to: Dr. Aysha N. Kinakool, Department of Psychology, York University, Toronto, Canada. E-mail: ayshak@yorku.ca

ORCID ID: https://orcid.org/0000-0001-8702-2568 
Tn addition to motor theories, the cerebellum is 1 believed to contribute to the execution of non-motor tasks that correspond to cognitive functions. ${ }^{1-4} \mathrm{With}$ the emergence of neuro-imaging techniques, these theories were supported by showing correlated activation patterns of the cerebral cortex and the contralateral cerebellum in a range of cognitive tasks. ${ }^{5-7}$ It was revealed that the cerebral cortex and cerebellum have mirrored network patterns where each part of the cerebrum (motor and cognitive) is represented by an area in the cerebellum. ${ }^{8}$ Several techniques also identified the presence of distinct cognitive closed-loop circuits that do not overlap with motor circuits of the cerebellum. ${ }^{9,10}$ Moreover, it was observed that not all patients with cerebellar stroke have motor impairments, highlighting that cerebellar motor syndromes are only associated with lesions in the cerebellar sensorimotor domains of function. ${ }^{11}$ This suggested that the cerebellum has a unique functional topography for influencing the performance of different cognitive functions.

In terms of cognitive functions and specifically attention, Petersen et $\mathrm{al}^{12}{ }^{12}$ illustrated the presence of 3 distinct but inter-connected networks for attention processing. It was concluded based on a multitude of evidence in the literature, that attention networks are anatomically distinct entities that are not linked to other pathways responsible for input, processing, or output of stimuli. The first network known as the alerting network, is responsible for arousal and vigilance systems where a warning cue activates phasic alteration in a person's resting state. ${ }^{12}$ It represents a tonic alertness where the appearance of a stimulus is followed by a response. The second network or orienting network focuses on filtering the information received to selectively focus on the spatial attributes of a signal, location or sensory stimulus. ${ }^{12}$ The third network known as the network of executive control, represents the ability to consciously focus on a target while simultaneously inhibiting interference from stimuli across other sensory systems. It is a state where a target or sensory stimulus is detected amidst the interference of other targets or stimuli.

Transcranial direct current stimulation (tDCS) is a non-invasive modulation method where different brain regions are stimulated below the threshold of neuronal damage. ${ }^{13,14}$ Low intensity current modulates brain regions, and this leads to the excitation or inhibition of different functions through the process of long-term

Disclosure. Authors have no conflict of interests, and the work was not supported or funded by any drug company. potentiation (LTP). ${ }^{15}$ With the emergence of these techniques, several studies have employed tDCS to investigate the effect of different polarities of current (anode, cathode and sham) on the functioning of the cognitive domain. ${ }^{16,17}$ Based on the concept of cerebellar brain inhibition (CBI), it has been proven that the cerebellum imposes an inhibitory effect through the facilitation of Purkinje fibers. ${ }^{18}$ This indicates that anodal stimulation facilitates the inhibitory effect of the Purkinje, while cathodal stimulation inhibits that effect and thereby increases excitability.

In general, very few studies have investigated the effect of cerebellar modulation on attention networks. Some studies have observed a modulation effect of the dorsolateral prefrontal cortex (DLPFC) on all attention tasks, while others revealed a modulatory effect of tDCS on the network of executive control alone. ${ }^{17,19}$ Ferrucci et $\mathrm{al}^{20}$ also assessed cerebellar modulation using cerebellar transcranial direct current stimulation (ctDCS) and observed improvement in a task that represented the network of executive control. The most recent and relevant study was conducted by Mannarelli et al, ${ }^{21}$ where the attention network test (ANT) was used to assess the effect of ctDCS on the functioning of the 3 attention networks. Findings revealed a significant difference in scores of the network of executive control. In the study paradigm, 7 cue conditions were administered to differentiate between the 3 attention networks. While the functioning of attention networks overlaps in daily life situations, they function separately along the dorsal and ventral visual streams. ${ }^{12}$ Moreover, the cerebellum has been proven to have a more profound effect on tasks that impose a higher cognitive load or difficulty. ${ }^{22,23}$ Therefore, the paradigm failed to precisely account for distinction between the alerting and orienting networks and overlooked that the orienting network can be sub-divided into several cue conditions that impose a higher mental load or difficulty.

Therefore, this study investigated the effect of different polarities of ctDCS on the efficiency of the 3 attention networks. A modified study paradigm was employed to individually assess the 3 attention networks and to investigate the efficiency of functioning of the orienting network based on different cue conditions with varying difficulty. Considering the concept of $\mathrm{CBI}$, anodal current was hypothesized to deteriorate the efficiency of the 3 attention networks. Contrarily, cathodal current was hypothesized to improve the same variables while sham current had no modulatory effect on the 3 attention networks.

Methods. Literature was extracted using the Summon library database of Imam Abdulrahman Bin 
Faisal University (IAU), Dammam, Kingdom of Saudi Arabia (KSA) and other databases like PubMed, and the Cochrane Library.

A sham-controlled, single-blind study where participants were randomized into 3 homogenous intervention groups: anode, cathode, and sham (10 females and males per group; Figure 1). All experiments took place at the neuroscience lab of IAU between January-July of 2018. Initially, 63 subjects underwent an assessment for eligibility and 3 were excluded as per the inclusion/exclusion criteria. A sample size calculator determined the total number of participants recruited in the study. Sixty participants were included based on the calculated Cohen's d (0.8 - large effect size), and the participants were divided into 3 sets of 20 per group.

A sample of convenience of 30 healthy males and 30 healthy females between 18-40 years of age were included (mean age $\pm S D ; 28.35 \pm 6.62$ ). The exclusion criteria included any nervous system disease (central or peripheral), psychiatric disorders, pregnancy, diabetes or prior participation in brain stimulation techniques. Before commencing the experiment, all participants provided written consent and all protocols complied

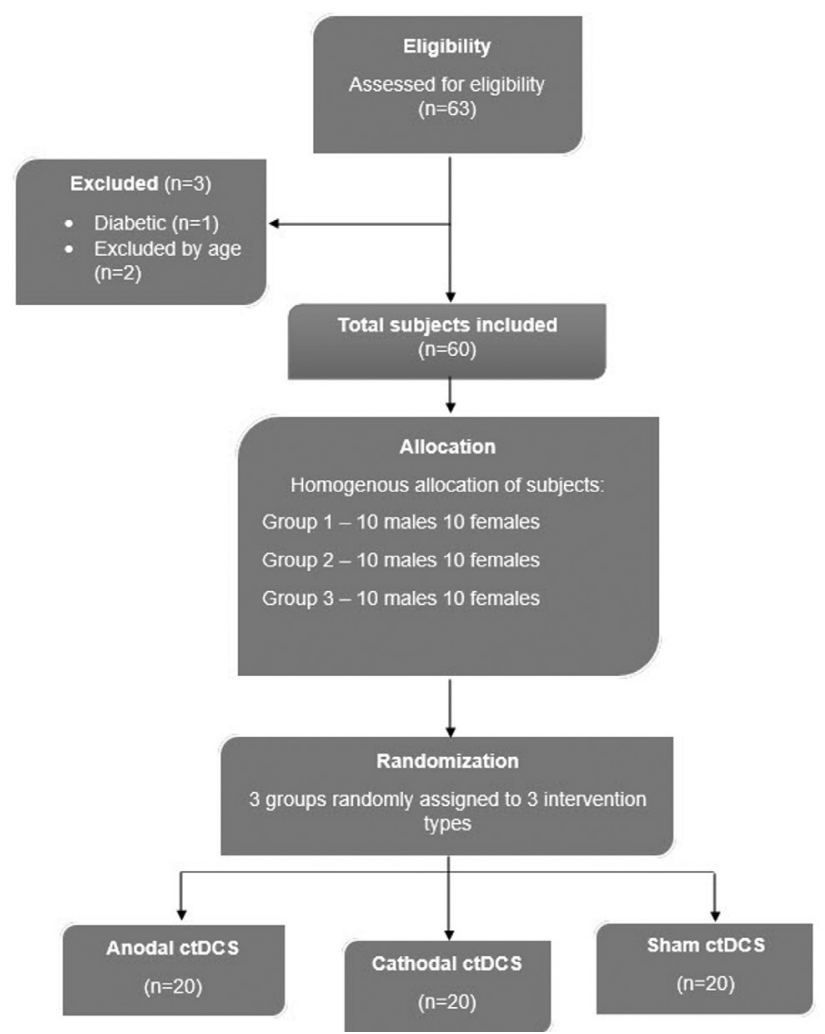

Figure 1 - Flow chart illustrating the participant randomization process, inclusion criteria and allocation. with the principles of the Declaration of Helsinki. The IAU institute review board (IRB) reviewed and approved the commencement of the study (IRB-PGS2017-03-174).

Randomization. Eligible participants were randomly assigned with a ratio of $1: 1: 1$ to the anode $(n=20)$, cathode $(n=20)$ and sham $(n=20)$ ctDCS intervention groups based on gender. The randomization sequence was conducted using www.randomizer.org to ensure random and equal gender distribution. To ensure blinding, the allocation was revealed only after the assessment and intervention were carried out. ${ }^{24}$

Intervention - cerebellar transcranial direct current stimulation (ctDCS). A direct current stimulator (Magstim Co., Whitland, UK) was used to administer ctDCS. The 10-20 EEG system was used as a reference guide for saline soaked electrode $(7 \times 5 \mathrm{~cm})$ placement. The active electrode was placed $2 \mathrm{~cm}$ inferior to the inion and the reference electrode was placed on the deltoid muscle of the dominant hand. ${ }^{20} \mathrm{~A}$ single stimulation session was conducted for 20 mins for all the groups. As per our previous study, a $2 \mathrm{~mA}$ current was administered, with $0.057 \mathrm{~mA} / \mathrm{cm}^{2}$ density and a resultant total charge of $1.14 \mathrm{C} / \mathrm{cm}^{2}{ }^{24}$ The same current intensity was ramped up for the initial 30 seconds and then ramped down to zero for the sham group to ensure blinding.

Outcome measures. All tasks were administered in the same sequential order for all subjects irrespective of the group they were assigned to. Prior to stimulation, the Edinburgh handedness inventory-short form determined the participants' hand dominance. ${ }^{25}$ Mental fatigue levels were assessed verbally from $0-10$ on the visual analogue scale (rest was to be given if fatigue was reported above 5/10). All outcomes were administered using the PEBL psychological test battery. ${ }^{26}$

A vigilance task was used to assess the role of the cerebellum in executing functions attributed to the efficiency of the alerting network. ${ }^{12,27}$ Subjects were presented with a warning cue before the appearance of a stimulus (Figure 2a). The subjects had to respond by pressing an assigned button as fast as possible only when a stimulus appeared on the screen. The outcome for the task was the mean reaction time (RT) in milliseconds (ms). Two RT scores V1-V2, corresponding to the efficiency of alerting attention were recorded for analysis. There was a total of 7 practice trials and 10 main trials in the task.

Posner's spatial orienting task assessed the role of the cerebellum in modulating the efficiency of the orienting network. This task assessed the ability to prioritize the location of a target stimulus by orienting attention. ${ }^{28}$ Subjects were asked to fix gaze on a centralized “+” sign 
and press the left or right shift key as fast as possible depending on where the "X" stimulus appeared (left or right to the "+") (Figure $2 \mathrm{~b}$ ). The main trials were divided into 4 blocks with 50 trials in each block $(200$ total trials). The mean RT of individual blocks was recorded in ms as P1, P2, P3 and P4. Similarly, the mean RT for the total number of trials, valid cues RT, invalid cues RT and neutral cues RT were recorded for analysis.

Finally, the Flanker task assessed the role of the cerebellum in modulating the efficiency of the network of executive control by focusing on a stimulus while simultaneously inhibiting detection of other stimuli. ${ }^{29}$ A row of 5 randomly directed arrows was presented in the center of the screen. Subjects were instructed to disregard the arrows in the periphery (namely, flankers) and focus on the central arrow (Figure 2c). A practice block of 8 trials was performed by the subjects before the main experimental block which included 16 trials. For analysis, the overall mean RT, congruent trials mean RT and incongruent trials mean RT were recorded (ms). The difference between incongruent and congruent trials RT, also known as "congruence cost" was also recorded.

Statistical analysis. Statistical analysis was conducted using the Statistical Package for the Social Science (SPSS) software version 25.0 (IBM Corp., Armonk, NY, USA). Data from the practice trials of tasks was not included for analysis. To ensure homogeneity of variances, the Levene's test was conducted. For alerting, a split-plot repeated measures (RM) ANOVA assessed within-subject differences in RT between the 2 trials V1 and V2 of each group $(p<0.05)$. Moreover, Group*Task interaction using one-way ANOVA was computed to assess the between-subjects mean RT scores. Post-hoc Tukey test analyzed multiple comparisons between groups.

In terms of orienting attention, RM split-plot ANOVA assessed the within-subject effect of the 4 blocks (P1, P2, P3 and P4) for the ctDCS groups. One-way ANOVA for Group*Task interactions (betweensubjects) was conducted for valid cues RT, invalid cues RT, neutral cues RT and mean RT. Concurrent with previous tasks, a post-hoc Tukey test analyzed multiple group comparisons.

Finally, one-way ANOVA assessed the Group*Task interaction of the Flanker task which represented the network of executive control. The between-subjects factor was analyzed for congruent trials RT, incongruent trials RT, congruence cost and the mean RT. Post-hoc Tukey test analyzed multiple comparisons between the groups.

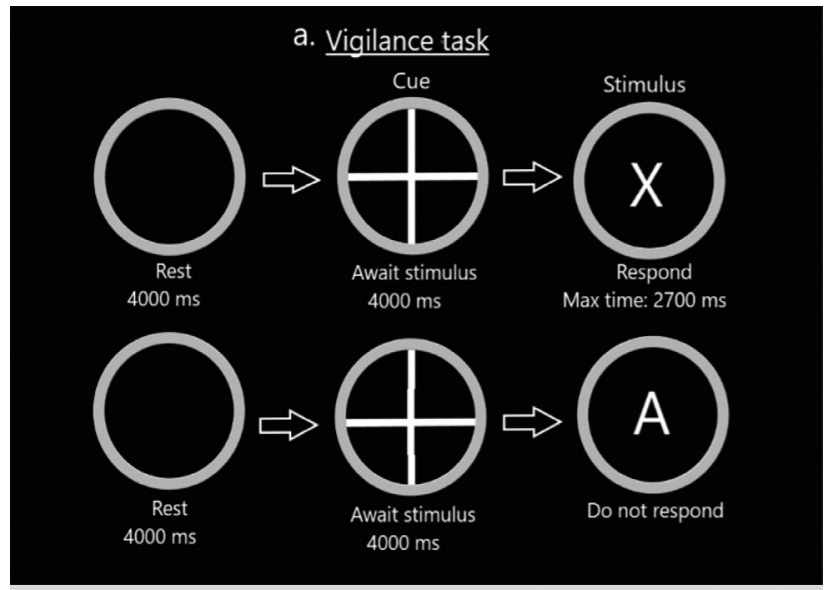

b. Posner's attention task
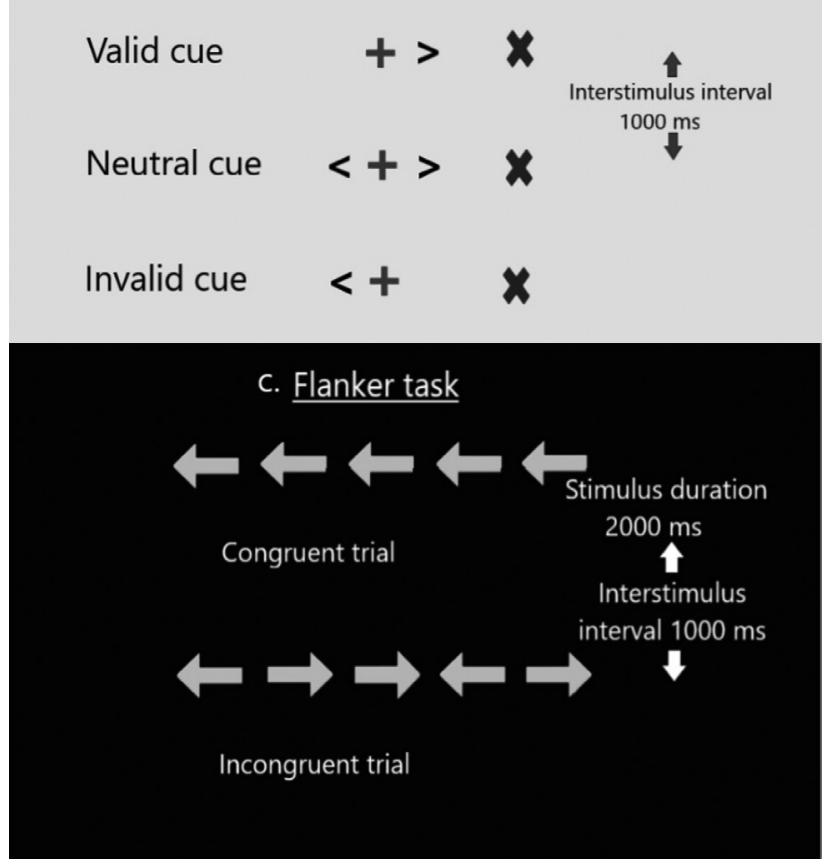

Figure 2 - Illustration of vigilance task for assessment of the alerting network of attention (a). Posner's attention task for assessment of the orienting network of attention (b). Flanker task for assessment of the network of executive control (c).

Results. Table 1 illustrates the demographic characteristics of the included participants.

Alerting network. Between-subjects ANOVA for Group*Task interaction of mean RT revealed a highly significant effect ( $\mathrm{F}[2,57]=6.64 ; p=0.003)$. Post-hoc Tukey test showed a highly significant effect of sham $(646.55 \pm 135.66)$ in comparison to cathode (525.05 $\pm 75.95 ; p=0.003)$. Similarly, sham scores were significantly higher than anode scores $(545.55 \pm 118.40$; 
$p=0.017)$. On the contrary, anode and cathode showed no significant difference between the mean RT scores (Figure 3). RM split-plot ANOVA conducted for the within-subjects factor of the 2 blocks (V1 and V2) revealed a non-significant Block*Group effect (Partial Eta squared - 0.059).

Orienting network. The between-subjects Group*Task effect was analyzed for valid cues RT, invalid cues RT, neutral cues RT and mean RT. Results revealed a non-significant mean RT Group*Task interaction between anode $(374.10 \pm 26.23)$, cathode $(368.82 \pm 11.97)$ and sham $(373.17 \pm 20.03)$ scores (F $[2,57]=0.386 ; p=0.682)$. Similarly, neutral cues RT Group*Task interaction revealed non-significant between-subjects effect for anode (397.00 \pm 68.95$)$, cathode $(424.60 \pm 80.22)$ and sham $(460.65 \pm 137.28)$ scores $(\mathrm{F}[2,57]=2.035 ; p=0.140)$. On the contrary, Group*Task interaction for valid cues RT scores revealed a highly significant between-subjects difference $(\mathrm{F}[2,57]=5.618 ; p=0.006$; Figure 4). Similarly, the Group*Task interaction for invalid cues RT betweensubjects revealed a significant difference $(F[2,57]=$ 4.868; $p=0.011)$. Figure 4 graphically demonstrates the mean scores of valid cues RT, invalid cues RT and neutral cues RT.

Table 1 - Demographic information of subjects in ctDCS groups $(n=20)$.

\begin{tabular}{lccc}
\hline Variables & \multicolumn{3}{c}{ Group } \\
& Anode ctDCS & Cathode ctDCS & Sham ctDCS \\
\hline Age (mean \pm SD) years & $28.3 \pm 6.12$ & $29.1 \pm 7.34$ & $27.65 \pm 6.61$ \\
Female gender n $(\%)$ & $10(50)$ & $10(50)$ & $10(50)$ \\
$\begin{array}{l}\text { Laterality right- } \\
\text { handed n (\%) }\end{array}$ & $17(85)$ & $18(90)$ & $18(90)$ \\
\end{tabular}

ctDCS - cerebellar transcranial direct current stimulation

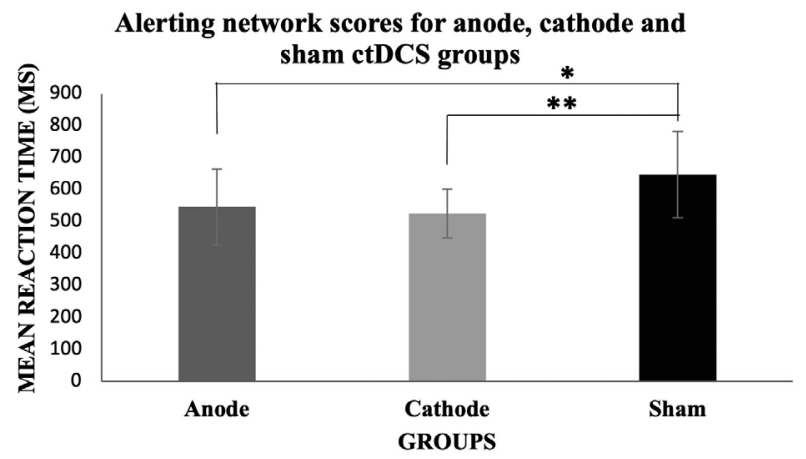

Figure 3 - Graph representing mean reaction times of the cerebellar transcranial direct current stimulation (ctDCS) groups for vigilance task; ${ }^{* *} p<0.01 ;{ }^{*} p<0.05$.
Post-hoc Tukey test was conducted for multiple comparisons of valid and invalid cues RT scores. Comparisons showed a significant difference of sham $(447.75 \pm 82.75)$ and anode $(399.15 \pm 53.60)$ valid cues RT scores $(p=0.043)$. Moreover, there was a highly significant difference between sham and cathode $(384.80 \pm 43.62)$ valid cues RT scores $(p=0.006)$. However, anode and cathode scores were not significantly different. In terms of invalid cues RT scores, post-hoc results revealed a significant difference in the scores of cathode and sham groups $(p=0.011)$. However, there was no significant difference between cathode and anode as well as anode and sham (Figure 4).

A split-plot RM ANOVA analyzed the withinsubjects Block*Group effect. The within-subjects Block*Group interaction showed a non-significant effect (partial eta squared - 0.054). Multiple pairwise block comparisons revealed a non-significant difference between the 4 (P1, P2, P3 and P4) blocks.

Network of executive control. There was no significant

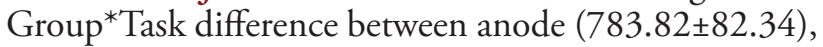
cathode $(801.95 \pm 0.46)$ and sham $(802.22 \pm 0.42)$ for congruent trials $(\mathrm{F}[2,57]=0.984 ; p=0.380)$. Similarly, incongruent trials showed no significant difference between anode $(786.73 \pm 69.84)$, cathode $(802.16 \pm 0.40)$ and sham $(802.17 \pm 0.39)$ groups $(F[2,57]=0.976$; $p=0.383)$. Also, there was no significant Group*Task difference between anode $(2.88 \pm 12.50)$, cathode $(0.21 \pm 0.45)$ and sham $(-0.05 \pm 0.37)$ for the congruence cost scores $(F[2,57]=1.011 ; p=0.370)$. Finally, the mean RT scores between anode $(784.11 \pm 81.20)$, cathode $(802.19 \pm 0.28)$ and sham $(802.18 \pm 0.28)$ were not significant $(\mathrm{F}[2,57]=0.991 ; p=0.378]$.

Discussion. Experimental findings revealed a polarity-independent Group*Task effect of anode

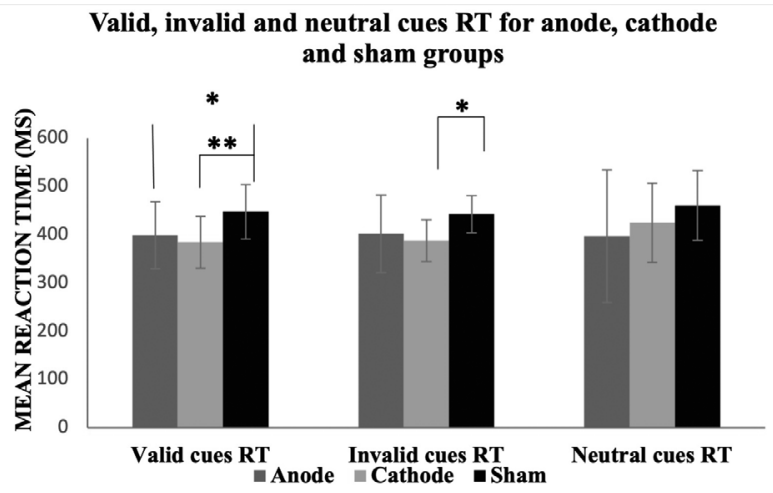

Figure 4 - Graph representing reaction times of anode, cathode and sham groups for valid cues RT, invalid cues RT and neutral cues RT of Posner's spatial orienting task; ${ }^{* *} p<0.01$; ${ }^{*} p<0.05$. 
and cathode in the efficiency of the alerting network. However, Cathodal stimulation seemed to modulate the cerebellum more significantly as compared to anode. This is not surprising as previous research has shown a similar effect of different tDCS polarities in functional domains. ${ }^{29-32}$ An enhancement in vigilance of an air traffic task was previously seen following anodal stimulation to the DLPFC. ${ }^{33}$ It was concluded that the decrease in RT was attributed to an increase in hemispheric blood oxygenation which was reflected by an improvement in alerting attention. Another study investigated the effect of stimulation of the inferior frontal cortex by examining the role of modulation in altering the attention networks. ${ }^{34}$ Anodal tDCS enhanced object detection performance, an attribute of the alerting network, without revealing an effect on the orienting or executive control networks. Since this study showed the involvement of cerebellar modulation in alerting, this could indicate the involvement of the cerebellum in a closed loop network with the frontal cortex to modulate alerting. Moreover, repeated measures pairwise comparison between the 2 blocks revealed no significant difference. This indicates that modulation occurred at the same pace between blocks and there was no significant difference in effect at the beginning or end of the task.

In terms of the orienting network, this study revealed no significant results for the Group*Task interaction of mean RT and neutral cues RT. These findings coincide with the findings of a recent study that revealed no modulatory effect of ctDCS on orienting attention. ${ }^{21} \mathrm{To}$ further analyze the efficiency of the orienting network based on cognitive load, valid and invalid cues RT were recorded in this study. The valid cues RT showed a significant Group*Task polarity-independent effect where cathode and anode both showed a significant stimulation effect on cerebellar modulation. Similarly, invalid cues RT also showed a highly significant Group*Task effect of cathodal ctDCS. These findings are justifiable as it has been previously shown that cerebellar stimulation showed a more notable effect when the difficulty of tasks was increased. ${ }^{22,23}$ This might be attributed to the ability of the cerebellum to adjust errors or improve performance when the cognitive load or difficulty is increased. This is reasonable as healthy subjects have high functioning cognitive processes and will demonstrate higher potential for improvement in performance when exposed to more difficult tasks (namely, invalid and valid cues).

In a previous attempt to investigate the effect of tDCS of different target sites on orienting attention, stimulation of the DLPFC, left and right parietal cortices revealed that the right posterior parietal cortex (PPC) had a modulatory effect on spatial reorienting. ${ }^{35}$ Similarly, Bolognini et $\mathrm{al}^{36}$ explored the influence of online tDCS applied over the PPC in improving orienting attention. The complex study paradigm comprised of a covert and an overt visual task and results showed a significant improvement in both forms of visual reorienting. In terms of the polarity, results of this study opposed a previous study that investigated the role of cortical stimulation on orienting attention. ${ }^{37}$ Stimulation of the intraparietal sulcus (IPS) showed an effect of cathodal tDCS on spatial reorienting while anodal tDCS had no effect. It was hence concluded that the inhibitory outcome of cathodal stimulation might be restricted to the motor and not cognitive domain. The fact that this study showed no improvement in the overall efficiency of orienting attention (illustrated by the mean RT) highlights that this network may not be affected by cerebellar attention processes.

This study revealed a non-significant Group*Task effect for the congruent trials, incongruent trials, congruence cost and mean RT of the flanker task. These findings indicate that cerebellar modulation using ctDCS had no effect on the network of executive control. Silva et al, ${ }^{17}$ previously studied attention dysfunction and assessed the effect of tDCS on the prefrontal cortex in modulating the 3 attention networks. By using the ANT as an outcome, active stimulation improved performance of orienting and executive control networks. Concurrently, a similar study employed ANT to investigate the effect of left prefrontal tDCS on attention. ${ }^{16}$ Results revealed a greater executive control (EC) following active anodal stimulation as compared to sham. Zmigrod et al, ${ }^{19}$ also demonstrated an effect of right DLPFC on stimulus conflict which corresponds to EC. Therefore, the findings of this study may indicate that the network of executive control is predominantly influenced by frontal cortex and that the modulation of the cerebellum does not contribute to its functioning.

Limitations. A limitation of this study is that a singleblind, single session of online ctDCS was conducted and so the cumulative effect of multiple sessions was not investigated. Moreover, the recruited participants were a healthy sample of convenience.

In conclusion, the results of the study indicate that the cerebellum has a specific role in modulating attention networks. Our findings revealed a significant polarity-independent effect of ctDCS on the efficiency of alerting attention despite previous findings suggesting that ctDCS had a modulatory effect on the network of executive control. ${ }^{21}$ There is a possibility that online stimulation of the cerebellum in this study prompted a 
different effect on the efficiency of attention networks as opposed to the offline stimulation used in other studies. We also observed and highlighted that while the cerebellum does not elicit an overall effect on the orienting network, it has a more profound effect on attention tasks with a higher cognitive load. This was demonstrated with significant polarity-independent effects on the valid cues RT scores and polaritydependent effects on invalid cues RT scores.

Future directions should aim to compare healthy subjects and patients with attention deficit disorders. The findings of this study indicate that ctDCS can be used as a treatment for patient populations with impaired attention processes. By identifying specific deficits in the functioning of attention networks, a more targeted approach can be implemented to treat patients with clinical manifestations of impaired attention.

Acknowledgment. The authors gratefully acknowledge all the participants who contributed to this study and Editage English Editing Services (www.editage.com) for English Language editing.

\section{References}

1. Leiner HC, Leiner AL, Dow RS. Does the cerebellum contribute to mental skills? Behav Neurosci 1986; 100: 443-454.

2. Leiner HC, Leiner AL, Dow RS. Reappraising the cerebellum: what does the hindbrain contribute to the forebrain? Behav Neurosci 1989; 103: 998-1008.

3. Leiner HC, Leiner AL, Dow RS. Cognitive and language functions of the human cerebellum. Trends Neurosci 1993; 16: 444-447.

4. Leiner HC. Solving the mystery of the human cerebellum. Neuropsychol Rev 2010; 20: 229-235.

5. Krienen FM, Buckner RL. Segregated fronto-cerebellar circuits revealed by intrinsic functional connectivity. Cereb Cortex 2009; 19: 2485-2497.

6. O’Reilly JX, Beckmann CF, Tomassini V, Ramnani N, Johansen-Berg H. Distinct and overlapping functional zones in the cerebellum defined by resting state functional connectivity. Cereb Cortex 2010; 20: 953-965.

7. Bernard JA, Seidler RD, Hassevoort KM, Benson BL, Welsh RC, Wiggins JL, et al. Resting state cortico-cerebellar functional connectivity networks: a comparison of anatomical and selforganizing map approaches. Front Neuroanat 2012; 6: 31.

8. Buckner RL. The cerebellum and cognitive function: 25 years of insight from anatomy and neuroimaging. Neuron 2013; 80: 807-815.

9. Kelly RM, Strick PL. Cerebellar loops with motor cortex and prefrontal cortex of a nonhuman primate. J Neurosci 2003; 23: 8432-8444.

10. Bostan AC, Dum RP, Strick PL. Cerebellar networks with the cerebral cortex and basal ganglia. Trends Cogn Sci 2013; 17: 241-254.

11. Schmahmann JD, Macmore J, Vangel M. Cerebellar stroke without motor deficit: clinical evidence for motor and non-motor domains within the human cerebellum. Neuroscience 2009; 162: 852-861.
12. Petersen SE, Posner MI. The attention system of the human brain: 20 years after. Annu Rev Neurosci 2012; 35: 73-89.

13. McCreery DB, Agnew WF, Yuen TG, Bullara L. Charge density and charge per phase as cofactors in neural injury induced by electrical stimulation. IEEE Trans Biomed Eng 1990; 37: 996-1001.

14. van Dun K, Bodranghien FC, Mariën P, Manto MU. tDCS of the cerebellum: where do we stand in 2016? technical issues and critical review of the literature. Front Hum Neurosci 2016; 10 : 199.

15. Stagg CJ, Nitsche MA. Physiological basis of transcranial direct current stimulation. Neuroscientist 2011; 17: 37-53.

16. Miler JA, Meron D, Baldwin DS, Garner M. The effect of prefrontal transcranial direct current stimulation on attention network function in healthy volunteers. Neuromodulation 2018; 21: 355-361.

17. Silva AF, Zortea M, Carvalho S, Leite J, Torres IL, Fregni F, et al. Anodal transcranial direct current stimulation over the left dorsolateral prefrontal cortex modulates attention and pain in fibromyalgia: randomized clinical trial. Sci Rep 2017; 7: 135.

18. Ugawa Y, Day BL, Rothwell JC, Thompson PD, Merton PA, Marsden CD. Modulation of motor cortical excitability by electrical stimulation over the cerebellum in man. $J$ Physiol 1991; 441: 57-72.

19. Zmigrod S, Zmigrod L, Hommel B. Transcranial direct current stimulation (tDCS) over the right dorsolateral prefrontal cortex affects stimulus conflict but not response conflict. Neuroscience 2016; 322: 320-325.

20. Ferrucci R, Brunoni AR, Parazzini M, Vergari M, Rossi E, Fumagalli M, et al. Modulating human procedural learning by cerebellar transcranial direct current stimulation. Cerebellum 2013; 12: 485-492.

21. Mannarelli D, Pauletti C, Currà A, Marinelli L, Corrado A, Delle Chiaie $\mathrm{R}$, et al. The cerebellum modulates attention network functioning: evidence from a cerebellar transcranial direct current stimulation and attention network test study. Cerebellum 2019; 18: 457-468.

22. Schlerf JE, Galea JM, Bastian AJ, Celnik PA. Dynamic modulation of cerebellar excitability for abrupt, but not gradual, visuomotor adaptation. J Neurosci 2012; 32: 11610-11617.

23. van Wessel BW, Claire Verhage M, Holland P, Frens MA, van der Geest JN. Cerebellar tDCS does not affect performance in the N-back task. J Clin Exp Neuropsychol 2016; 38: 319-326.

24. Kinakool AN, Abualait TS. Online polarity-dependent effects of cerebellar transcranial direct current stimulation on motor speed and fine manual dexterity. A randomized controlled trial. Saudi Med J 2020; 41: 18-24.

25. Veale JF. Edinburgh handedness inventory - short form: a revised version based on confirmatory factor analysis. Laterality 2014; 19: 164-177.

26. Piper BJ, Mueller ST, Geerken AR, Dixon KL, Kroliczak G, Olsen RH, et al. Reliability and validity of neurobehavioral function on the psychology experimental building language test battery in young adults. PeerJ 2015; 3: e1460.

27. Langner R, Eickhoff SB. Sustaining attention to simple tasks: a meta-analytic review of the neural mechanisms of vigilant attention. Psychol Bull 2013; 139: 870-900.

28. Diamond A. Executive functions. Annu Rev Psychol 2013; 64: 135-168. 
29. Ferrucci R, Marceglia S, Vergari M, Cogiamanian F, MrakicSposta S, Mameli F, et al. Cerebellar transcranial direct current stimulation impairs the practice-dependent proficiency increase in working memory. J Cogn Neurosci 2008; 20: 1687-1697.

30. Shah B, Nguyen TT, Madhavan S. Polarity independent effects of cerebellar tDCS on short term ankle visuomotor learning. Brain Stimul 2013; 6: 966-968.

31. Antal A, Terney D, Poreisz C, Paulus W. Towards unravelling task-related modulations of neuroplastic changes induced in the human motor cortex. Eur J Neurosci 2007; 26: 2687-2691.

32. Orban de Xivry JJ, Marko MK, Pekny SE, Pastor D, Izawa J, Celnik P, et al. Stimulation of the human motor cortex alters generalization patterns of motor learning. J Neurosci 2011; 31: 7102-7110.

33. Nelson JT, McKinley RA, Golob EJ, Warm JS, Parasuraman R. Enhancing vigilance in operators with prefrontal cortex transcranial direct current stimulation (tDCS). Neuroimage 2014; 85: 909-917.
34. Coffman BA, Trumbo MC, Clark VP. Enhancement of object detection with transcranial direct current stimulation is associated with increased attention. BMC Neurosci 2012; 13 : 108.

35. Roy LB, Sparing R, Fink GR, Hesse MD. Modulation of attention functions by anodal tDCS on right PPC. Neuropsychologia 2015; 74: 96-107.

36. Bolognini N, Fregni F, Casati C, Olgiati E, Vallar G. Brain polarization of parietal cortex augments training-induced improvement of visual exploratory and attentional skills. Brain Res 2010; 1349: 76-89.

37. Moos K, Vossel S, Weidner R, Sparing R, Fink GR. Modulation of top-down control of visual attention by cathodal tDCS over right IPS. J Neurosci 2012; 32: 16360-16368.

\section{Statistics}

Excerpts from the Uniform Requirements for Manuscripts Submitted to Biomedical Journals updated November 2003. Available from www.icmje.org

Describe statistical methods with enough detail to enable a knowledgeable reader with access to the original data to verify the reported results. When possible, quantify findings and present them with appropriate indicators of measurement error or uncertainty (such as confidence intervals). Avoid relying solely on statistical hypothesis testing, such as the use of $P$ values, which fails to convey important information about effect size. References for the design of the study and statistical methods should be to standard works when possible (with pages stated). Define statistical terms, abbreviations, and most symbols. Specify the computer software used. 